\title{
Can ICON replace IOTN and PAR?
}

\author{
A comparison of the Index of Complexity Outcome and Need (ICON) with the Peer Assessment Rating (PAR) and the \\ Index of Orthodontic Treatment Need (IOTN) N. A. Fox, C. Daniels and T. Gilgrass Br Dent J 2002; 193: 225-230
}

\section{Aim}

To evaluate any relationship between ICON, IOTN and PAR. To establish whether or not ICON could replace these indices as a measure of orthodontic treatment complexity, outcome and need.

\section{Method}

The study models of 55 consecutively treated cases were examined and PAR, IOTN and ICON recorded.

\section{Results}

The study showed significant correlations between IOTN and ICON with respect to need and PAR and ICON with respect to outcome.

\section{Conclusion}

It appears that ICON does reflect UK opinion and the current study provides some evidence that ICON may effectively replace PAR and IOTN as a means of determining need and outcome.

\section{IN BRIEF}

- This paper investigates a new orthodontic occlusal index, the Index of Outcome, Complexity and Need (ICON). It suggests that this single index can replace the PAR index and the Index of Orthodontic Treatment Need (IOTN). The implications are:

- The single index (ICON) which is easier to learn and use than PAR and IOTN could measure orthodontic treatment need and treatment standards.

- The index could be easily incorporated into the orthodontic assessment of a patient in general dental practice.

- The inclusion of IOTN in the general dental services has been proposed. ICON would be quicker to learn, apply and more information could be gleaned than just treatment need.

\section{COMMENT}

IOTN and PAR were developed in the late 1980s to address the contentious issues of need for orthodontic treatment and quality of outcome.

Both indices have their critics, yet most orthodontists acknowledged them as the best tools available for monitoring samples of caseloads objectively. Unfortunately, to assess need and outcome, two scoring protocols had to be learnt. Furthermore, neither index was appropriate for assessment of treatment complexity, which still relied on clinical judgement.

As the authors note, one criticism of PAR was its leniency towards certain inappropriate or incomplete treatments. Arguably this was because it evolved as a peer assessment tool, validated against the opinions of dentists with varying orthodontic experience, when many British GDS treatments still involved only removable appliances. Although pragmatic and sensitive, this was perhaps unlikely to promote higher standards as readily as validation against expert opinion. The weightings also vary nationally, which hinders the comparison of data internationally.

ICON promises an answer to some of these problems. As a unified index of complexity, need and outcome, calibrated against an international panel of orthodontists from Europe and America, its standards should be more appropriate to modern orthodontics worldwide. However, PAR and IOTN are now established within the orthodontic psyche, so will we make the switch to ICON for audit and research? Part of this question lies in perceived parity - is 'definite need' under ICON equivalent to 'definite need' under IOTN? Is a 'great improvement' equivalent to the same under PAR or does ICON represent a totally different set of standards?

The authors address this issue by comparing the correlation of ICON with PAR and IOTN over a sample of 55 cases, and they discuss some mathematical and interpretational differences between the indices. They conclude that ICON could replace IOTN, and that it bears a close relationship to PAR, whilst being more stringent in its requirements for the 'greatly improved' category.

How easily will the profession adopt the new index, though? Inevitably, established users of PAR and IOTN will consider having to learn a new index irksome. Moreover, will our managers and paymasters accept it? Whilst the salaried services embraced PAR and IOTN quite readily, it has taken a decade for IOTN to be accepted as a threshold for treatment in the English and Welsh GDS. Despite the apparent advantages of ICON, therefore, there may be a lengthy changeover period, during which all three indices will co-exist.

Dr Liz Turbill Senior Dental Officer in Orthodontics, Central Manchester PCT, and P/T Honorary Lecturer in Orthodontics, University Dental Hospital of Manchester. 